\title{
Role of Lithium lodide Addition to Lithium Thiophosphate: Implications beyond Conductivity
}

Nikhilendra Singh ${ }^{\dagger}$, James P. Horwath ${ }^{\ddagger}$, Patrick Bonnick ${ }^{\dagger}$, Koji Suto $^{+1}$, Eric A. Stach ${ }^{\ddagger}$, Tomoya Matsunaga $^{+1}$, John Muldoon ${ }^{\dagger}$ and Timothy S. Arthur ${ }^{*+}$

†Toyota Research Institute of North America, 1555 Woodridge Ave, Ann Arbor, MI 48105

‡ Department of Materials Science and Engineering, University of Pennsylvania, 3231 Walnut Street, Philadelphia, PA 19104

Supporting Information

I. Detailed TEM Experimental Section

II. Figures 


\section{Detailed TEM Experimental}

- Specific to ex-situ TEM: All samples for ex-situ analysis were loaded onto $3 \mathrm{~mm}$ 300-mesh lacy carbon coated copper grids (Ted Pella) within an Argon atmosphere glove box $\left(<0.1 \mathrm{ppm} \mathrm{O}_{2}\right.$ and $\mathrm{H}_{2} \mathrm{O}$ ). The loading was carried out by directly scooping the grid through the sample material contained within a glass vial. As such, this was a dry-casting method and no solvents were utilized during the process at any time to prevent possible reactions between sample materials and solvents. The materials cast and analyzed using this process were $\mathrm{Li}_{3} \mathrm{PS}_{4}$ and $\mathrm{Li}_{3} \mathrm{PS}_{4}$ :0.5 LiI. In addition to general imaging and elemental analysis, ex-situ TEM was utilized as a benchmark to establish the necessary microscope settings (e.g. probe size, beam current, spot size, exposure time, etc.) to successfully image and analyze the materials without damage from the beam itself. It should be noted that each material is expected to have different behavior under the beam and as such should be benchmarked prior to in-situ TEM analysis efforts to ensure an optimized setup. Below, we provide a description of how the ex-situ holder (JEOL single-tilt) was setup for the experiments highlighted in this manuscript:

○ The holder was brought into the glove box using standard protocols and the holder tip was disassembled within the glove box to load a designated sample material. Specific tools for the holder were also brought into the glove box to ensure damage-free disassembly/assembly of the holder.

- For all experiments, $\mathrm{Li}_{3} \mathrm{PS}_{4}$ or $\mathrm{Li}_{3} \mathrm{PS}_{4}: 0.5 \mathrm{LiI}$ materials were loaded onto the grid using the procedure described above. The material loaded grid was then assembled into the holder component.

○ The reassembled holder was now secured for air-free transfer from the glove box by using a customized air-free holder cap. Once secure with this cap, the holder was removed from the glove box using standard protocols and immediately moved to the TEM for loading.

- The holder was loaded into the TEM while the air-lock for the TEM holder vented either nitrogen or argon gas to ensure air-free entry of the holder into the TEM. The custom airfree holder cap was removed from the holder at the TEM point of entry immediately prior to loading the holder into the TEM and pumping it down instantly to minimize sample exposure to air during this process. The total time that the sample was exposed to air was on the order of 5 to 10 seconds.

- Specific to in-situ TEM: All sample analysis was carried out using a Biasing Nanomanipulator Holder designed by Hummingbird Scientific. The holder assembly was carried out within an Argon atmosphere glove box $\left(<0.1 \mathrm{ppm} \mathrm{O}_{2}\right.$ and $\left.\mathrm{H}_{2} \mathrm{O}\right)$. This in-situ holder utilized a $3 \mathrm{~mm} 300$ - 
mesh lacy carbon coated copper half-grid (Ted Pella) and a tungsten (W) scanning tunneling microscopy (STM) probe (Bruker). The biasing capability of the in-situ holder allowed for the application and observation of a current and/or voltage between the half-grid and STM probe. The movable STM probe could be brought into contact with the half-grid to complete a circuit and conduct electrochemistry experiments within the TEM. Below, we provide a description of how the in-situ holder was setup for the experiments highlighted in this manuscript:

- The holder was brought into the glove box using standard protocols and the holder tip was disassembled within the glove box to separate components for the half-grid and the probe. Specific tools (procured from Hummingbird Scientific) for the holder were also brought into the glove box to ensure damage-free disassembly/assembly of the holder.

○ For all experiments, $\mathrm{Li}_{3} \mathrm{PS}_{4}$ or $\mathrm{Li}_{3} \mathrm{PS}_{4}: 0.5 \mathrm{LiI}$ materials were loaded onto the half-grid using the procedure described for ex-situ TEM. The material loaded half-grid was then assembled into the holder component designed for the half-grid.

- The $\mathrm{W}$ probe was converted into a lithium $(\mathrm{Li})$ coated $\mathrm{W}$ probe for our applications, as follows. Li metal foil (MTI Corporation) was first cleaned by mechanical scraping of the foil surfaces using the plastic cap of a standard $20 \mathrm{ml}$ glass vial (VWR). This scraped Li foil piece was placed on top of a 100-micron thick nickel (Ni) foil (Alfa Aesar) which was contained within a glass petri dish (VWR). The petri dish, along with the $\mathrm{Ni}$ and $\mathrm{Li}$ foil materials were then heated using a hot plate until the $\mathrm{Li}$ was observed to melt at which point the $\mathrm{W}$ probe was very gently dipped into the molten $\mathrm{Li}$ to procure the $\mathrm{Li}$ coated $\mathrm{W}$ probe. The dipping was accomplished by bringing the molten Li into contact with the probe, not vice-versa, to ensure safety of the fragile probe.

- The holder was now completely reassembled within the glove box and the holder tip (containing the material loaded half-grid and Li probe components) was secured for airfree transfer from the glove box by using a customized air-free holder cap. Once secure with this cap, the holder was removed from the glove box using standard protocols and immediately moved to the TEM for loading.

- The holder was loaded into the TEM while the air-lock for the TEM holder vented either nitrogen or argon gas to ensure air-free entry of the holder into the TEM. The custom airfree holder cap was removed from the holder at the TEM point of entry immediately prior to loading the holder into the TEM and pumping it down instantly to minimize sample exposure to air during this process. The total time that the sample was exposed to air was on the order of 5 to 10 seconds. 
Once the holder was pumped down in the TEM, a suitable $\mathrm{Li}_{3} \mathrm{PS}_{4}$ or $\mathrm{Li}_{3} \mathrm{PS}_{4}: 0.5 \mathrm{LiI}$ sample was located on the edge of the half-grid closest to the Li probe, and the Li probe was moved into position to contact this sample using manual and electronic adjustments to the probe via Hummingbird Scientific software. Upon contact, the noted in-situ TEM electrochemical experiments in the manuscript were carried out using a Bio-Logic SP-200 Potentiostat. 


\section{Figures}
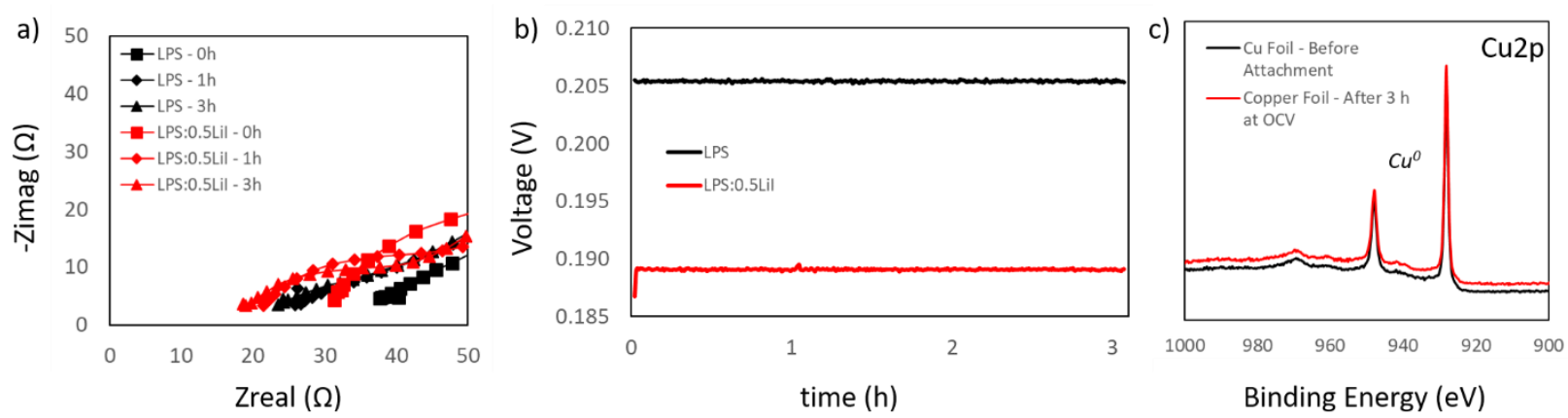

Figure S1. Evidence for Cu/LPS and Cu/LPS:0.5LiI stability before cell equilibrium. a) EIS spectra of a Cu/LPS or LPS:0.5 LiI/Li half-cell after cell fabrication $(0 \mathrm{~h})$, after $1 \mathrm{~h}$ and after $3 \mathrm{~h}$ of heating from room temperature to $60 \mathrm{\circ}$. b) $0 \mathrm{CV}$ measurements showing stable cell voltages before lithium metal deposition. c) XPS Cu2p spectra of a copper foil surface before cell fabrication and after $3 \mathrm{~h}$ of OCV measurements in a Cu/LPS/Li half-cell.
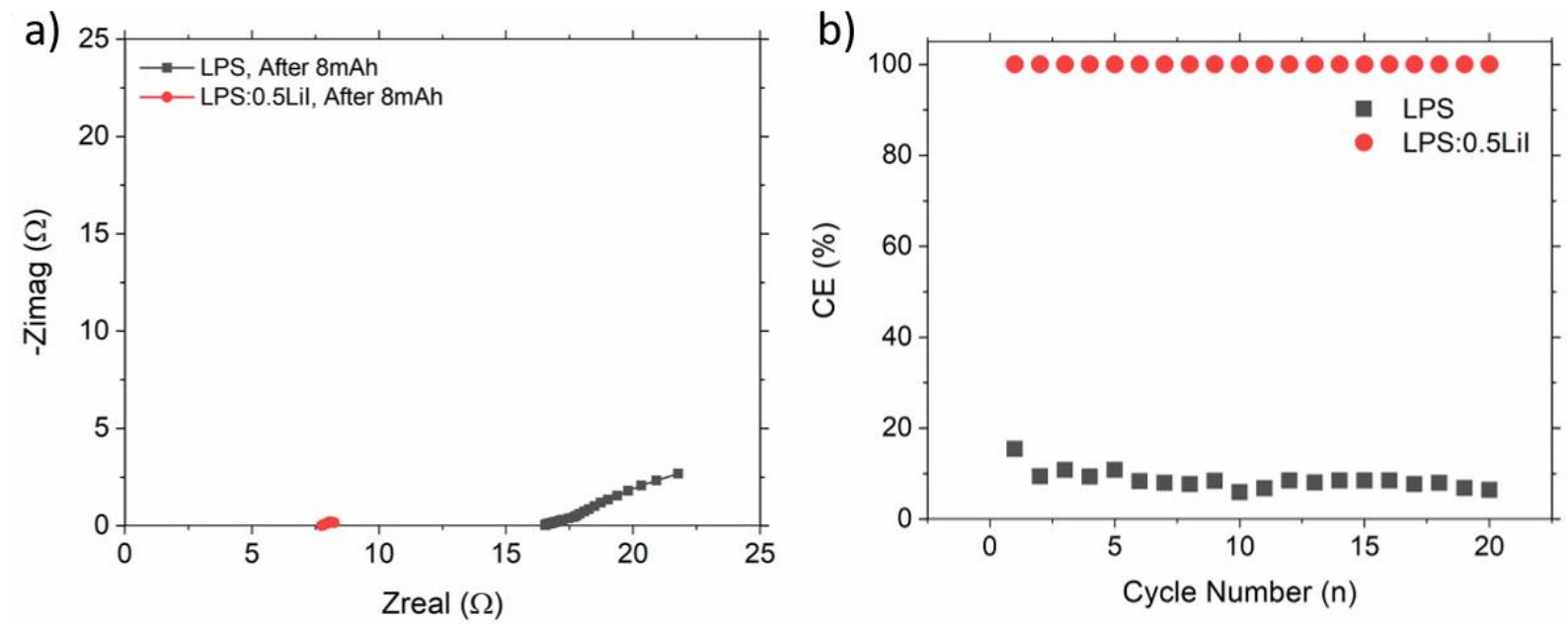

Figure S2. a) EIS of $\mathrm{Cu} / \mathrm{SE} / \mathrm{Li}$ half-cells after $8 \mathrm{~mA}$.h of galvanic lithium plating at $0.25 \mathrm{~mA} / \mathrm{cm}^{2}$. b) Coulombic Efficiency (CE), defined as $\left(Q_{\text {stripping }} / Q_{\text {plating }}\right)$, of $\mathrm{Cu} / \mathrm{SE} / \mathrm{Li}$ half-cells at $1 \mathrm{~mA} / \mathrm{cm}^{2}$ for $1 \mathrm{~mA}$.h. 


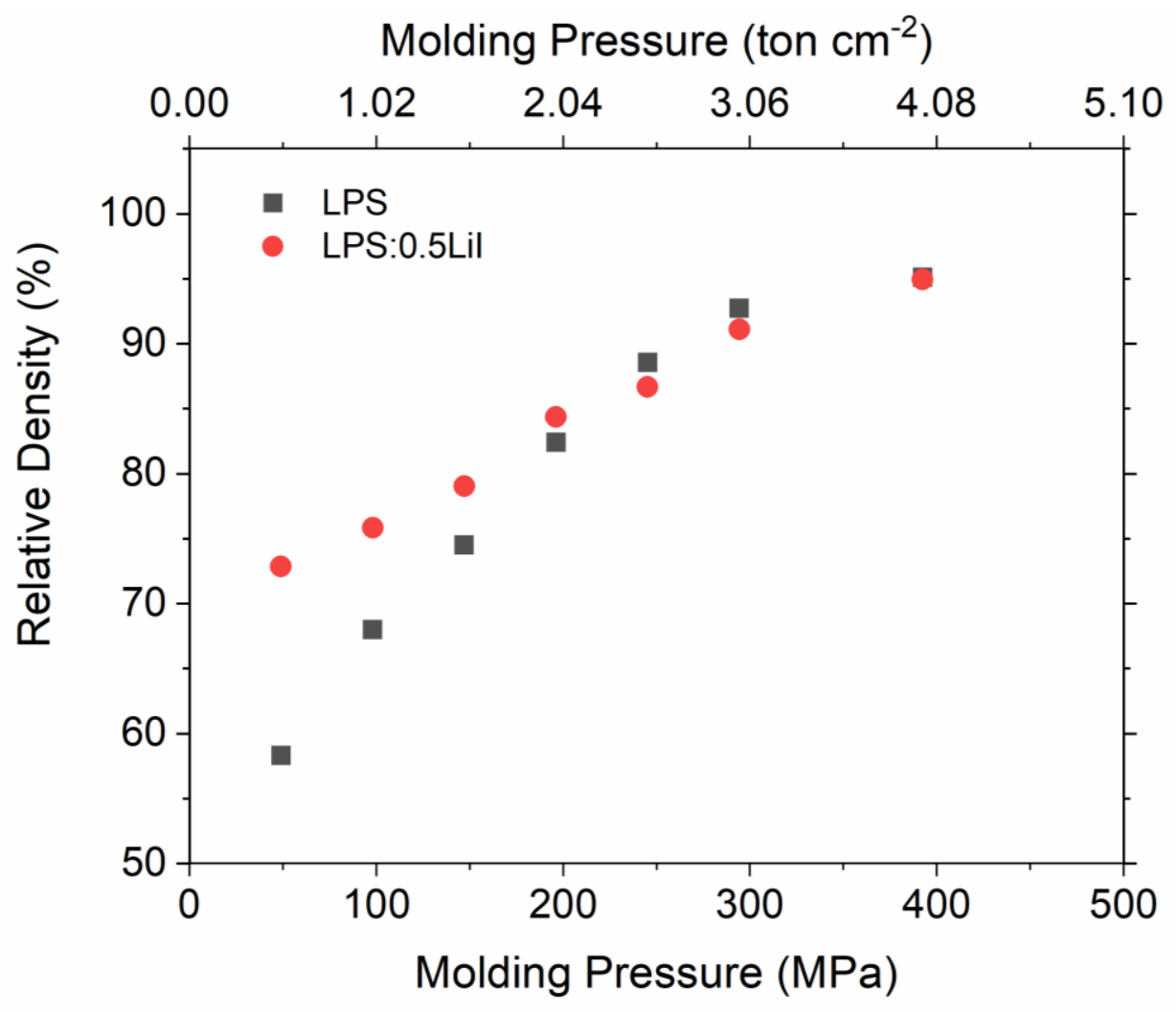

Figure S3. a) Relative density of pelletized solid electrolytes under increasing molding pressure. Relative density is calculated from $D_{m} / D_{F}$ where $D_{m}=$ geometrically measured density of a pellet and, $D_{F}=$ fully-densified material obtained by hot pressing solid-electrolytes according to Tatsumisago et al. (Ref. 9) $D_{F}=1.88 \mathrm{~g} / \mathrm{cm}^{2}$ for LPS and $D_{F}=2.33 \mathrm{~g} / \mathrm{cm}^{2}$ for LPS:0.5LiI.

Pristine

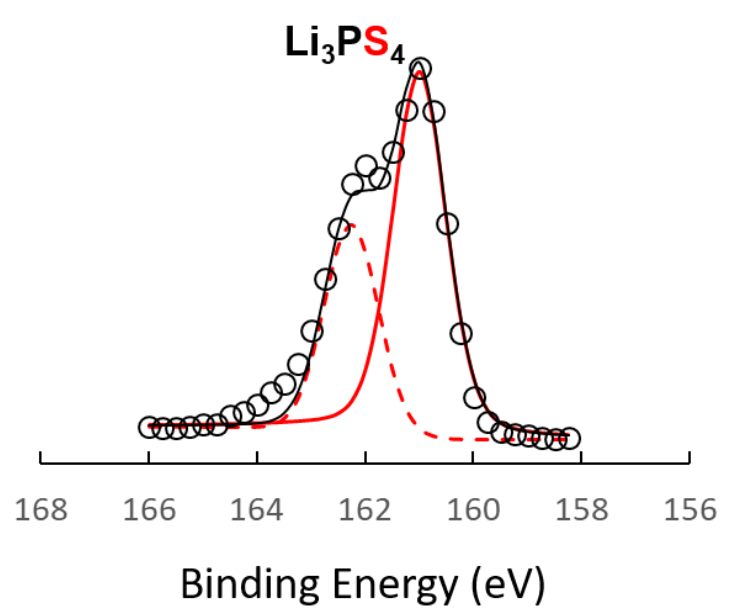

Cycled

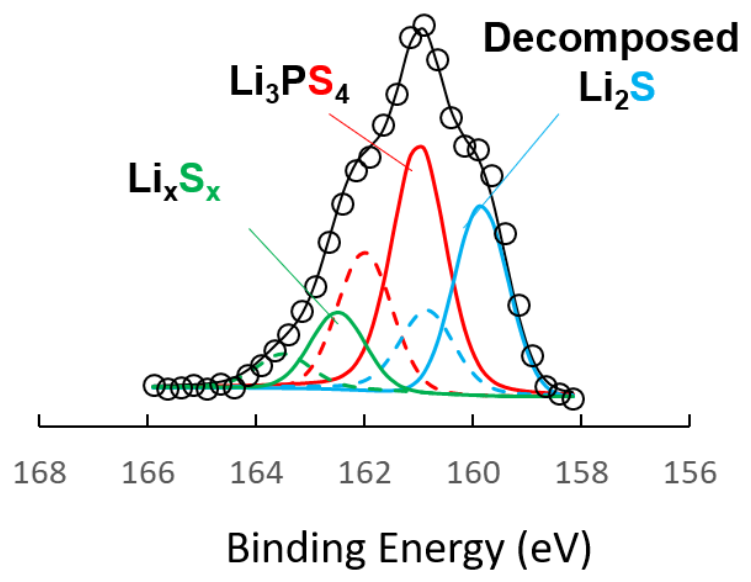

Figure S4.S2p XPS spectra and de-convolution of LPS:0.5LiI as a pristine pellet and after galvanic cycling with lithium metal. 

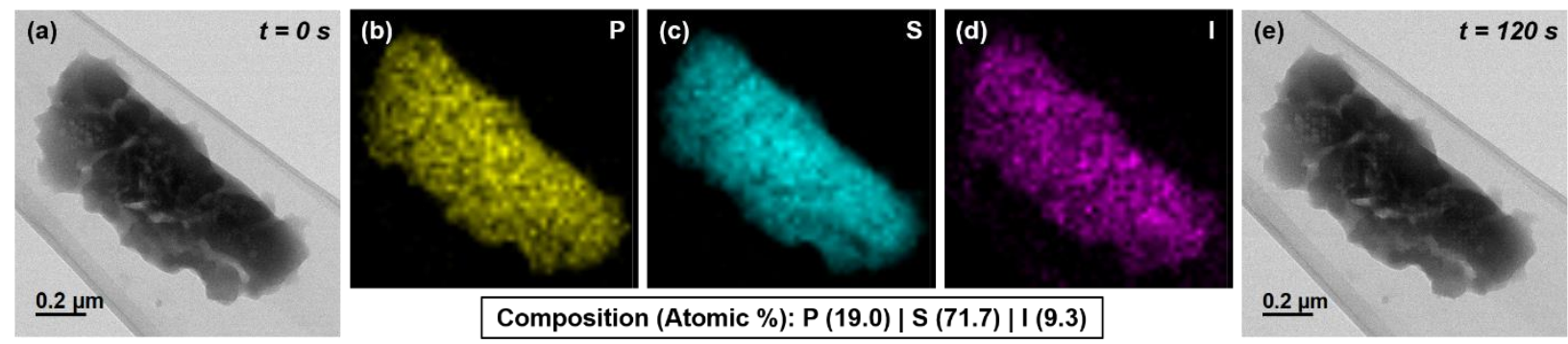

Figure S5. LPS:0.5LiI stability is shown for $120 \mathrm{~s}$ in a transmission electron microscope. Iodine is evenly distributed throughout the LPS:0.5LiI particle, indicating stability to low pressure conditions needed for TEM.
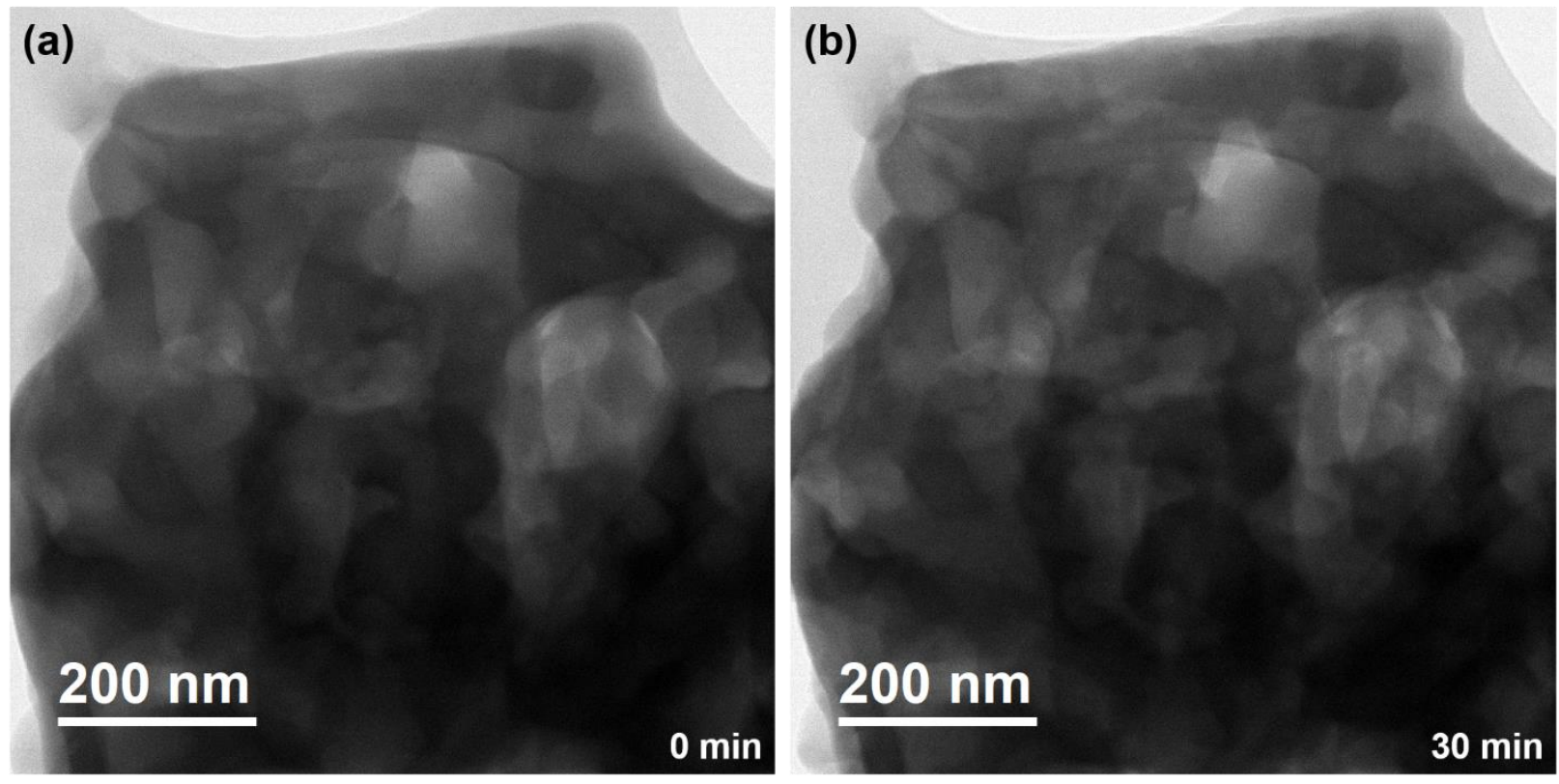

Figure S6. a)-b) Bright field STEM images of LPS:0.5Lil taken over a 30 minute timeframe to highlight the stability of LPS:0.5LiI under the electron beam due to the sample preparation procedures followed in this work. It should be noted that without the procedures being followed in this work, the same sample material would degrade under the electron beam within 2 minutes. 

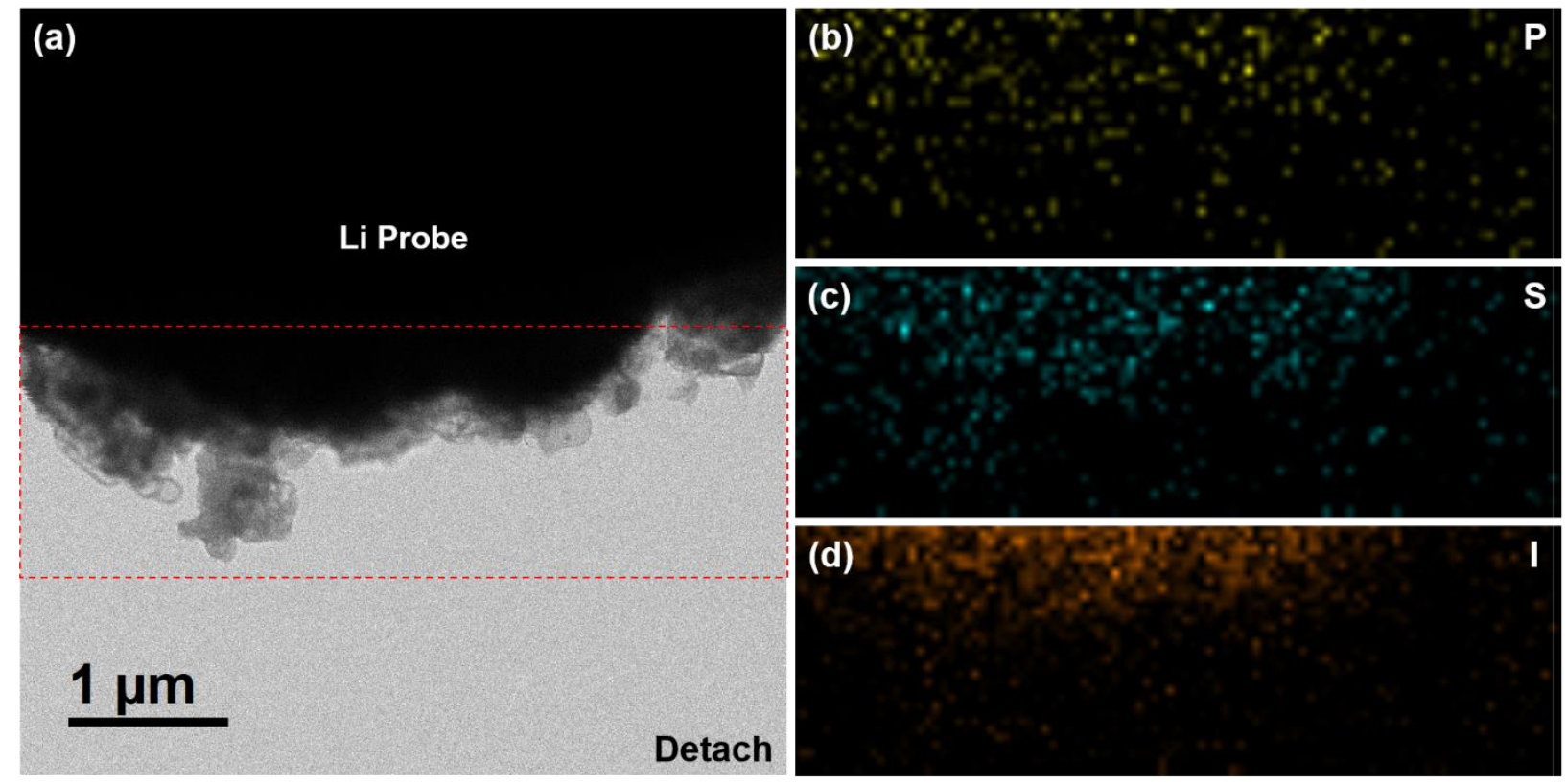

(d)

Figure S7. a) Bright field STEM image of a Li-metal probe detached after contact and electrochemical plating/stripping of Li from LPS:0.5LiI. b)-d) are EDS maps of P, S and I after detachment, respectively. The area of interest selected for the EDS maps is highlighted in a). 